\title{
A Comparison of Robotic Assisted Laparoscopic Prostatectomy and External Beam Radiation Therapy for Urinary and Sexual Function in Men Treated for Prostate Cancer
}

\author{
Julia Fischer*, Human Physiology
}

ABSTRACT

INTRODUCTION: Prostate cancer is the second most commonly diagnosed cancer in men. Robot Assisted Laparoscopic Prostatectomy (RALP) and External Beam Radiation Therapy (EBRT) are standard treatments for clinically localized prostate cancer, but both of these treatments have negative consequences for urinary and sexual function in patients.

PURPOSE: To compare changes in urinary and sexual function for men treated with RALP and EBRT.

HYPOTHESIS: It was hypothesized that patients treated with EBRT would have better recovery of sexual function, and patients treated with RALP would have better recovery of urinary function.

METHODS: Urinary and sexual function for patients treated for prostate cancer was examined using Expanded Prostate Index Composite (EPIC) questionnaires. These questionnaires were completed before treatment and two years after initial treatment in 32 men treated with EBRT, and 104 men treated with RALP. The difference between initial treatment scores and 2-year scores were analyzed with a general linear model(GLM) procedure to assess the quality of life outcomes for EBRT and RALP.

RESULTS: No significant difference was found for change in urinary function for either treatment group $(p=0.41)$. EBRT was found to significantly increase recovery sexual function compared to RALP $(p=0.04)$.

CONCLUSION: EBRT is a superior treatment for preserving sexual function in men with prostate cancer, whereas there is no significant difference in recovery of urinary function.

\footnotetext{
* Julia Fischer grew up in Hillsboro Oregon and graduated with a major in Human Physiology and minors in Biology and Chemistry from the University of Oregon in 2017. She interned for two years at Oregon Urology Institute (OUI) and conceived of this project with the help of OUI physicians and staff. Along with her internship with OUI, she was also involved in the Human Physiology department as a Learning Assistant and participated in the Oregon Marching Band as a Drum Major. Julia is interested in infectious disease and public health, and got to experience these interests while working with ANOVA Health Institute in Tzaneen South Africa during the summer of 2016. Julia aspires to be a physician specializing in pediatric infectious disease. This year she will be working as a medical scribe and applying to medical school, as well as continuing to teach as a Human Physiology Learning Assistant. Please direct correspondence to juliafischer3.14@gmail.com.
} 


\section{OVERVIEW OF PROSTATE CANCER ANATOMY AND PHYSIOLOGY OF THE PROSTATE}

The prostate is a male specific organ that encircles the urethra and borders the inferior aspect of the bladder (see fig.1). The prostate contains the ejaculatory duct, which is the end of the spermatic tube that extends all the way down from the testes. Sperm travel through this tube to arrive at the urethra during ejaculation. Nerves that supply the urethra and penis run posteriorlaterally along the prostate and are contained in the neurovascular bundles (see fig. 2). The function of the prostate is to create and secrete certain components found in semen. One of these components is prostate specific antigen (PSA), which is a protein that helps maintain a low viscosity in semen to allow the sperm to swim freely (Lee et al. 436).

\section{Prostate Cancer}

Prostate cancer is the second most frequently diagnosed cancer in the United States, falling only behind skin cancer (Haas et al. 1). In a healthy functioning prostate the PSA that is produced is almost entirely secreted into the urethra during ejaculation, with only small levels leaking into bloodstream circulation (Stenman et al. 1). When prostate cells become cancerous they rapidly proliferate and create an excess amount of PSA. This is more PSA than can be secreted into the urethra during ejaculation, and the excess is leaked into circulation and that be detected by a blood tests and indicate abnormal prostatic tissue growth (Smith et al. 29). Thus, PSA screenings are conducted by physicians in men over fifty to look for sharp increases in PSA that could indicate prostate cancer (Smith et al. 29).

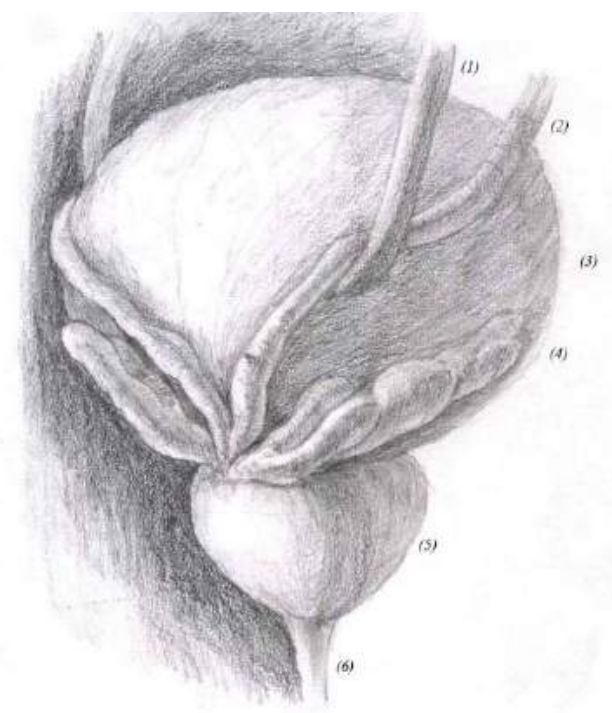

Fig 1. Anatomy of the Prostate (1) Ureter, (2) Vas Deferens, (3) Bladder, (4) Seminal Vesicle (5) Prostate (6) Urethra from Martin Allums;

2017

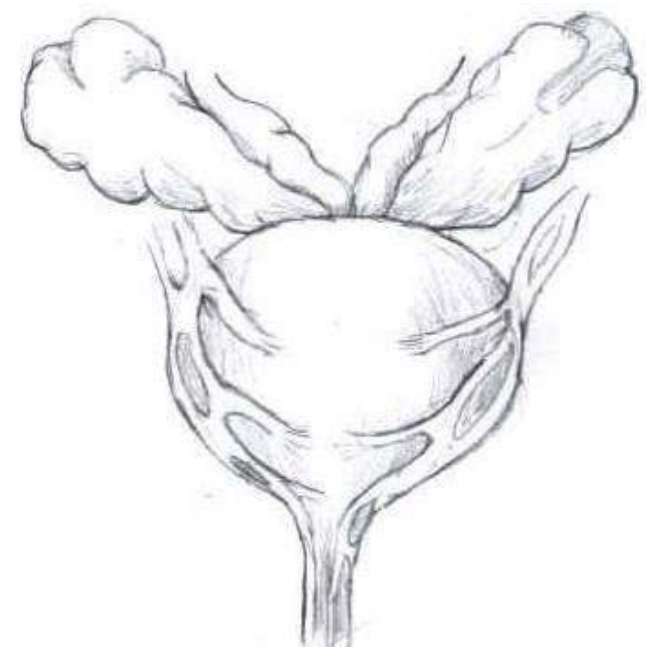

Fig 2. Neurovascular Bundles of the Prostate from Martin Allums; 2017 
While elevated PSA levels may indicate a cancer, there are other conditions that can cause PSA levels to rise. Benign Prostatic Hyperplasia (BPH) is a common condition among men over the age of fifty which can also results in elevated levels of PSA (Stenman et al. 1). BPH is a condition where the prostate grows larger without evidence of malignancy (Stenman et al. 1). This additional growth of prostate cells will also produce an excess of PSA, which will be detected in a blood test. Other conditions such as a bladder or prostate infection can also cause the PSA to spike. If no inflammation or infection of the prostate is clinically apparent, then further evaluation of elevated PSA includes Transrectal Ultrasound (TRUS) guided biopsy of the

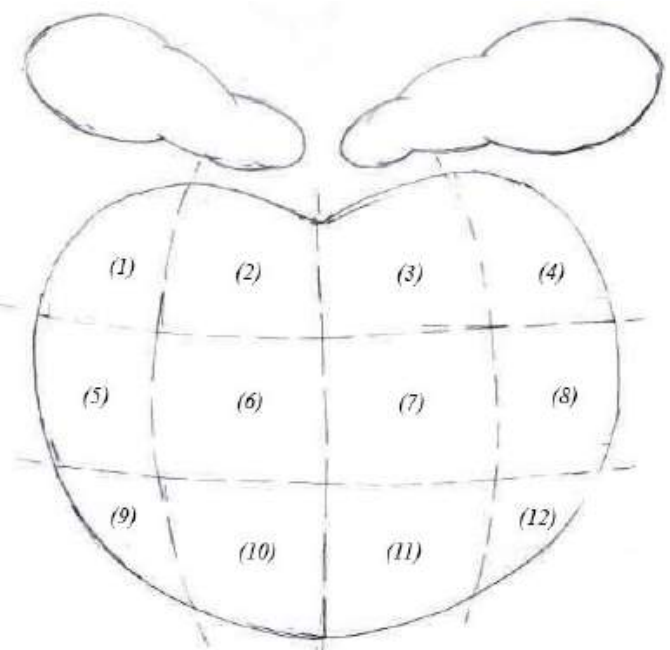

Fig 3. Transrectal Ultrasound Guided Biopsy Specimen Sites from Martin Allums; 2017 prostate to deduce if prostate cells have become cancerous (Heidenreich et al. 70).

A Transrectal Ultrasound (TRUS) guided biopsy takes up to twelve samples of prostatic tissue in each region of the prostate to test for malignancy (see fig. 3). The samples taken from the TRUS biopsy are examined and given a Gleason Score. The Gleason Score represents the number of cancerous cells within a sample and their histological appearance in an assigned grade from 1-5 (Humphrey 293). A grade of one represents cells that are packed closely together but are still separated with a uniform appearance and well differentiated growth patterns. A grade of five represents the most altered appearance of the cells with large, observable, different shaped masses (Humphrey 293). The grades 2-4 represent the range of appearances of the cancer cells between the grades 1 and 5 (Humphrey 293). The Gleason Score is calculated by summing the two largest grades assigned to the histological sample of the prostate tissue, generating a value of 2-10 (Humphrey 293). Patients with a Gleason Score of 2-6 are candidates for active surveillance, essentially close surveillance of PSA progression without definitive treatment to avoid over-treatment (Shah 1810). Patients with a Gleason Score of seven or greater are in need of definitive therapy (Shah 1810).

Another tool used to assess the extent of the cancer is the Tumor-Node-Metastasis (TNM) staging system. The TNM cancer staging system is used to assign a stage to cancerous prostatic samples (Edge et al. 1471). The TNM outlines the location of the cancer in relation to the prostate gland and the rest of the body. The $\mathrm{T}$ in the score denotes a tumor in the prostate, the $\mathrm{N}$ signifies a tumor in a lymph node, and the $M$ indicates metastasis in other locations in the body. A common score is T2a, which indicates a tumor involving one half a prostatic lobe or less. The TNM staging can be diagnosed from a TRUS biopsy, or by palpation of the prostate via rectal exam (Edge et al. 1417). 


\section{Treatment of Prostate Cancer}

\section{Definitive Treatment Options}

Surgical removal of the prostate is a common procedure used to treat prostate cancer. The surgical techniques of prostatectomies have evolved in the last two decades from the traditional Radical Retropubic Prostatectomy (RRP) which was considered the standard of care for treatment of prostate cancer (Coelho et al. 2003). A RRP is an invasive procedure which involves a large open incision in the abdomen and surgical dissection of the prostate (Barré 72). New surgical techniques have developed such that laparoscopic dissection (a minimally invasive procedure with a few small incisions) of the prostate can be achieved with the assistance of a da Vinci Robotic Surgical System. This new technology allows surgeons to perform a Robot Assisted Laparoscopic Prostatectomy (RALP) with minimal invasion compared to the open incision of the RRP (see fig. 4). RALP operations can be performed as

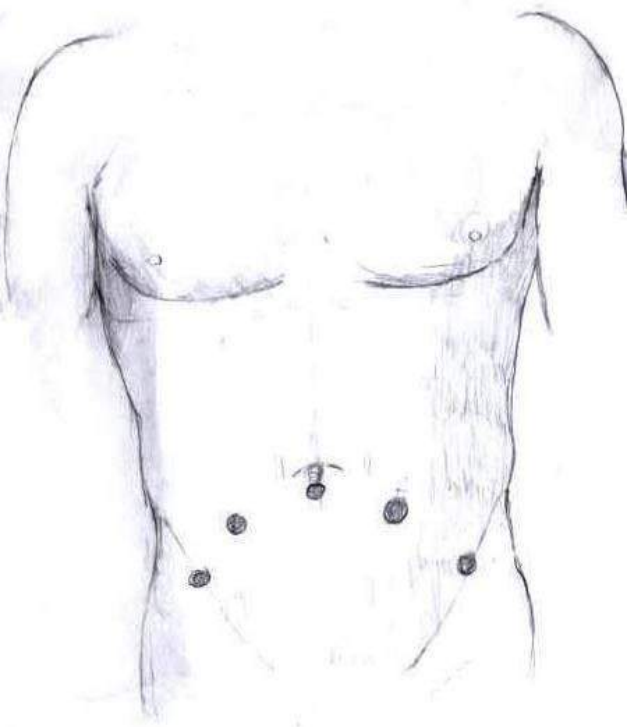
Nerve Sparing (NS) procedures, where the neurovascular

Fig 4. Surgical Incision Sites for RALP from Martin Allums; 2017 bundles are dissected away from the prostate in an attempt to preserve the urinary and sexual function of the patient (Coelho et al. 2006). As there is a neurovascular bundle that runs on both sides of the prostate, a NS procedure can be bilateral, where both neurovascular bundles are dissected away, or unilateral, if only one is dissected away. The type of NS procedure depends on the girth and location of the tumor. If the tumor extends into the regions of these neurovascular bundles, the neurovascular bundles will not be dissected away in an attempt to remove all cancerous cells (Talcott et al. 1117).

External Beam Radiation Therapy is another common treatment for prostate cancer. EBRT is radiation delivered from an external source directed at the prostate from different angles to preserve the tissue around the prostate (Heidenreich et al. 72). If a patient has received a prostatectomy as initial treatment and there is reoccurrence of prostate cancer, they can go on to receive EBRT (Heidenreich et al. 76). However, once a patient receives EBRT for their initial treatment, they have received a lifetime dose of radiation to that area and are not able to receive additional radiation treatment for their prostate cancer should it recur (Heidenreich et al. 76).

Another treatment for prostate cancer is Androgen Deprivation Therapy (also referred to as hormone therapy). The growth of most prostate cancer cells is dependent on androgens (sex hormones), most often testosterone or dihydrotestosterone (Miyamoto et al. 332). To treat prostate cancer, hormone deprivation therapy aims to stop the production of testosterone. This is achieved by either pharmaceutically or surgically castrating the patient, or stopping the body's natural production of androgens (Miyamoto et al. 332). Androgen deprivation therapy is not a 
curative treatment, it is used to slow the disease progression and extend patient life (Miyamoto et al. 344). This therapy is often used with patients whose initial treatment of RALP or EBRT has not been successful, resulting in the disease spreading to other areas of the body (Heidenreich et al. 76).

\section{Introduction}

Patients who undergo treatment for prostate cancer find it difficult to maintain a high quality of life due to impaired urinary and sexual function, since nerves that supply the urethra and penis are contained in neurovascular bundles that run posterior-laterally along the prostate (Walsh et al. 473). Surgical removal or radiation of the prostate can damage these nerves and have a severe impact on urinary and sexual function of patients after treatment, which can lead to a lower quality of life (Walsh et al. 473). Quality of life for patients being treated for prostate cancer is a major factor in the decision of which treatment to choose. Poorer quality of life due to incontinence and erectile dysfunction can lead to depression, poorer quality of sleep, and lower levels of overall of health (Coyne et al. 1388).

For cancer that is localized to the prostate, both Robot Assisted Laparoscopic Prostatectomy (RALP) and External Beam Radiation Therapy (EBRT) are considered acceptable treatments (Schreiber et al. 21). Patient input is essential in the treatment decision, thus randomizedcontrol studies are difficult to perform to determine which treatment has better quality of life outcomes. Researchers have heavily relied on retrospective studies to assess the benefits of one treatment versus another, but these studies often yield no clear distinction. EBRT and RALP are both associated with a decline in Health Related Quality of Life (HRQOL) directly after treatment, but thus far there is no clear indication as to which is the superior treatments in terms of the recovery of urinary and sexual function (Miller et al. 2775; Frank et al. 2151). Many studies that investigate this were performed prior to 2006, when RALP surgical techniques became more widespread, and therefore only included comparisons of Radical Retropubic Prostatectomy to EBRT. These studies found that patients treated with EBRT had better recovery of urinary and sexual function than those treated with RRP (Litwin et al. 2239; Potosky et al. 1358). The purpose of this study was to compare recovery of urinary and sexual function using information from the Prostate Cancer Health Related Quality of Life (HRQOL) database in patients that were treated with RALP or EBRT to determine if EBRT results in better sexual and/or urinary function recovery in patients two years after initial treatment compared to RALP. The study used the Expanded Prostate Index Composite (EPIC) questionnaire to measure the patients' urinary, bowel, hormonal, and sexual irritation. It was hypothesized that the changes in sexual function EPIC scores would indicate better sexual function recovery for patients treated with EBRT than RALP, and changes in urinary function EPIC scores would indicate better recovery of urinary function in patients treated with RALP than EBRT. 


\section{Methods}

\section{Patient Selection}

This study compared External Beam Radiation Therapy (EBRT) to Robot Assisted Laparoscopic Prostatectomy (RALP), so patients selected for this study were good candidates for both EBRT and RALP at the time of their initial treatment. Almost all patients are good candidates for EBRT, but not all qualify for RALP. Therefore, only EBRT patients that were specifically noted to also qualify for RALP were selected for this study (see table 1). Surgical guidelines denote that patients should live long enough to benefit from lack of malignancy. As RALP is an invasive procedure, a patient should have a life expectancy of 10 years or more to receive treatment

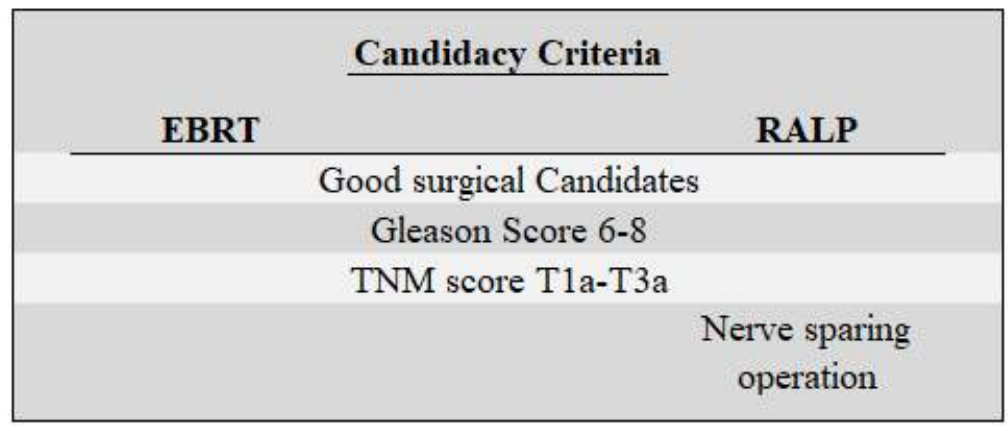

(Lepor 182). Although the probability that a man 70 years old will live 10 more years after

Table 1

Criteria for subject selection of each treatment group prostatectomy is about 58\% (Lepor 183), Oregon Urology Institute physicians do not discriminate treatment based on age and will select RALP if a patient has an estimated ten-year life expectancy. Thus, patients were selected for this study if it specified in their charts that they were good surgical candidates at the time of their initial treatment consultation. Patients selected had a Gleason Score of 6-8, which indicated that they needed initial treatment. Patients with a Gleason score of 9-10 are classified in the high-risk category and most often receive multiple treatment types such as surgery or radiation with hormone deprivation therapy (Fowler, Jr et al. 3221). Multiple treatments could confound the study results, so these patients were not included. Selected patients had a TNM score in the range of T1a-T3a, indicating they still had organ confined prostate cancer, and were therefore eligible for prostatectomy (Lepor 183). Patients who were selected for the RALP subject group underwent a form of a nerve sparing procedure (Bilateral, Right, Split, etc.) to improve erectile function.

\section{Data Collection}

\section{Sexual and Urinary Function Data}

Measurements for Sexual and Urinary function have been acquired through the EPIC. EPIC questionnaires inquire about the patient's urinary, bowel, hormonal, and sexual irritation, which reflects their urinary, bowel, hormonal, and sexual function (Wei et al. 899). The EPIC questionnaires relates the scores (1-5 or 1-4) in a section and calculates a percentage that gives a summary of the function for that section. Thus, if the top score is selected by the patient for each question in a section such as urinary function, that patient would receive 100 for that section 
when their EPIC score is calculated. Higher scores represent less irritation and better function. The scores recorded for sexual and urinary function were used in this study, and all other information was omitted. A packet with this questionnaire is sent out to patients willing to participate in the Prostate Cancer Database before their treatment, then quarterly for the first year following treatment, and then annually. The two-year time point was chosen to assess recovery of urinary and sexual function since improvement in both generally does not occur past twenty-four months (Penson et al. 42; Donovan et al. 1429).

\section{Treatment Related Information}

Many co-variants were included in this study. Smoking status was determined based on smoking status at time of treatment. Co-morbidities were recorded from conditions recorded in patients' chart at time of treatment. Race was recorded from self-reported race in patients' charts. Use of hormone therapy was determined from listed medications on the patient's chart. Erectile aid use before and at the two-year time point was determined based off medication lists and notes made in the patient's chart. Other information regarding Gleason Score and other biopsy information was recorded from the biopsy pathology report, and the TRUS surgical report. Surgery related information, such as procedures performed and diagnostics, were recorded from the prostatectomy pathology report and the surgical notes.

\section{Statistical Analysis}

The patients' clinical presentations were analyzed with Welch's t-test (two tailed t-test with unequal variance). Demographic characteristics, which were included as possible confounding variables, were analyzed with Fisher's exact test. A Wilcoxon rank-sum test was used to compare baseline BMI of patients in each treatment group, as there were not normal distributions. General Linear Models (GLM) were used to assess significant differences between treatment groups, and identify if any variables were confounding the relationship between treatment groups and EPIC scores for urinary and sexual function.

Table 2 shows the clinical presentations of each treatment group. The patients in both treatment groups were largely similar. One statistically significant difference was the TNM Stage between the two treatment groups. The RALP treatment group had a slightly higher average TNM staging of T2b than the EBRT group's T2a. 


\section{Patient Demographics}

\section{Table 2}

Comparison of Clinical Presentations for EBRT and RALP Patients

${ }^{*}$ Statistically significant with Welch's T-test $\mathrm{p}<.05$.

\begin{tabular}{|c|c|c|c|c|}
\hline & \multicolumn{2}{|c|}{ EBRT } & \multicolumn{2}{|c|}{ RALP } \\
\hline & Average & Mode & Average & Mode \\
\hline $\begin{array}{c}\text { Age at start of } \\
\text { tx (yr) }\end{array}$ & $\begin{array}{c}66 \\
\text { SD: } 7\end{array}$ & 59 & $\begin{array}{c}64 \\
\text { SD: } 6.8\end{array}$ & 68 \\
\hline $\begin{array}{l}\text { PSA Level } \\
\text { (ng/mL) }\end{array}$ & $\begin{array}{c}5.7 \\
\text { SD: } 3\end{array}$ & 5.5 & $\begin{array}{c}6.1 \\
\text { SD: } 4.3\end{array}$ & 5.5 \\
\hline $\begin{array}{l}\text { Prostate Size } \\
\qquad(\mathrm{mL})\end{array}$ & $\begin{array}{c}43 \\
\text { SD: } 15.8\end{array}$ & - & $\begin{array}{c}39.8 \\
\text { SD: } 20.2\end{array}$ & 33 \\
\hline Gleason Score & $\begin{array}{c}6.4 \\
\text { SD: } 0.5\end{array}$ & 6 & $\begin{array}{c}6.6 \\
\text { SD: } 0.7\end{array}$ & 6 \\
\hline 1st Grade & $\begin{array}{c}3.1 \\
\text { SD: } 0.3\end{array}$ & 3 & $\begin{array}{c}3.2 \\
\text { SD: } 0.4\end{array}$ & 3 \\
\hline 2nd Grade & 3.3 & 3 & 3.4 & 3 \\
\hline
\end{tabular}




\begin{tabular}{|c|c|c|c|c|}
\hline & SD: 0.5 & & SD: 0.5 & \\
\hline $\begin{array}{c}\text { Biopsies (\% of } \\
\text { positive } \\
\text { samples) }\end{array}$ & $\begin{array}{c}35.6 \\
\text { SD: } 22.4\end{array}$ & 25 & $\begin{array}{c}35 \\
\text { SD: } 20.4\end{array}$ & 33 \\
\hline $\begin{array}{l}\text { Highest } \% \text { of } \\
\text { Cores }\end{array}$ & $\begin{array}{c}49 \\
\text { SD: } 28.7\end{array}$ & 80 & $\begin{array}{c}46 \\
\text { SD: } 29.9\end{array}$ & 80 \\
\hline $\begin{array}{c}\text { Clinical TNM } \\
\text { Stage }^{*}\end{array}$ & $\mathrm{t} 2 \mathrm{a}$ & $\mathrm{t} 1 \mathrm{c}$ & $\mathrm{t} 2 \mathrm{~b}$ & t2c \\
\hline BMI & $\begin{array}{c}29.1 \\
\text { SD: } 4.4\end{array}$ & - & $\begin{array}{c}27.9 \\
\text { SD: } 3.9\end{array}$ & 25.9 \\
\hline
\end{tabular}

Fisher's exact test revealed significant differences in the amount of cardiac disease between treatment groups. The EBRT group had more instances of cardiac disease, with $41 \%$ of the patients with cardiac disease (see table 3). This prevalence of a disease, which influences the patient's overall health, suggests that the EBRT patient group was generally less healthy than the RALP group. Fisher's exact test revealed statistically significant differences in the number of patients treated with hormone therapy between groups during the time interval this study analyzed. The EBRT group had more patients treated with hormone therapy within the two-year interval post initial treatment (16\% vs. $1 \%$ ) (see table 3). As there is decreased libido, as well as other symptoms, related to the use of hormone therapy, the patient's sexual function EPIC scores could be affected by this treatment. The demographic categories of race and use of erectile aid were not included in the GLM analysis due to an unacceptable amount of missing data. 


\section{Table 3}

Comparison of Covariates in Treatment Patient Population

*Indicates statistically significant differences between the treatment groups $\mathrm{p}<.05 * *$ Indicates statistical significant differences between treatment groups $\mathrm{p}<.001{ }^{\circ}$ Indicates variable not included in analysis due to missing data

\begin{tabular}{|c|c|c|}
\hline & \multicolumn{2}{|c|}{ Number of Patients (\%) } \\
\hline & EBRT & RALP \\
\hline \multicolumn{3}{|c|}{ Patients Included } \\
\hline Total number of patients & 32 & 104 \\
\hline Lost to follow up & $0(0 \%)$ & $7(6.7 \%)$ \\
\hline Deceased & $0(0 \%)$ & $2(2 \%)$ \\
\hline Radiation after initial tx & $0(0 \%)$ & $10(10 \%)$ \\
\hline $\begin{array}{l}\text { Pt w/ family history of } \\
\text { prostate cancer }\end{array}$ & $11(34 \%)$ & $32(31 \%)$ \\
\hline \multicolumn{3}{|c|}{ Comorbidities $^{* *}$} \\
\hline No comorbidities & $12(38 \%)$ & $94(90 \%)$ \\
\hline Cardiac disease $^{* *}$ & $13(41 \%)$ & $2(2 \%)$ \\
\hline Hypertension & $1(3 \%)$ & $5(5 \%)$ \\
\hline Arterial disease & $0(0 \%)$ & $1(1 \%)$ \\
\hline
\end{tabular}




\section{Smoking Status*}

\begin{tabular}{crr} 
Pt never smoked & $12(38 \%)$ & $58(56 \%)$ \\
Pt current smoker & $2(6 \%)$ & $1(1 \%)$ \\
Pt former smoker & $18(56 \%)$ & $39(38 \%)$ \\
\hline Alaskan native & Race $^{\circ}$ & \\
White & $1(3 \%)$ & $0(0 \%)$ \\
Race not reported & $23(72 \%)$ & $65(63 \%)$ \\
\hline
\end{tabular}

\section{Erectile Aid Use ${ }^{\circ}$}

No Erectile aid use before tx

$6(19 \%)$

$92(88 \%)$

Erectile aid use before tx

$26(81 \%)$

$11(11 \%)$

Erectile aid use not reported before $t x$

$0(0 \%)$

$1(.01 \%)$

No Erectile aid after tx

Erectile aid use after tx

Erectile aid use not reported after $\mathbf{t x}$
$22(69 \%)$

$9(28 \%)$

$50(48 \%)$

$1(3 \%)$

$22(21 \%)$ 


\section{Hormone Therapy*}

Received hormone therapy

No hormone therapy
$5(16 \%)$

$27(84 \%)$
$1(1 \%)$

$103(99 \%)$

\section{Statistical Tests}

Figure 5 shows the distribution of urinary change scores for EBRT and RALP. The unadjusted GLM model illustrated in Table 4 had an overall $F$ value of 0.52 . This $F$ value is not less than o.05, suggesting that this model is not a successful fit, so an adjusted model was run.

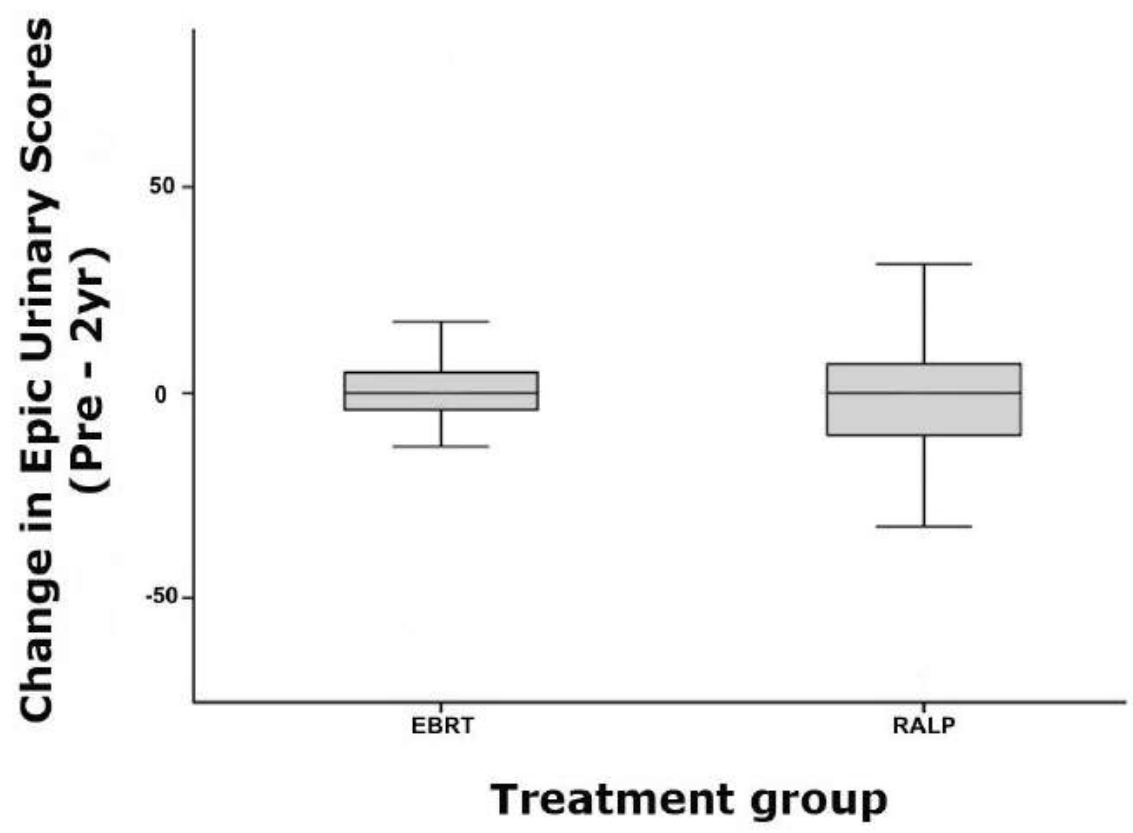

Fig 5. Distribution of Urinary EPIC Score Change

In this figure, zero denotes baseline. As two-year scores were subtracted from pre-treatment scores, negative scores indicate improvement from initial urinary function and positive scores represent a worsening from initial urinary function. 


\section{Table 4}

Reported Values from the Urinary Function Unadjusted GLM Model

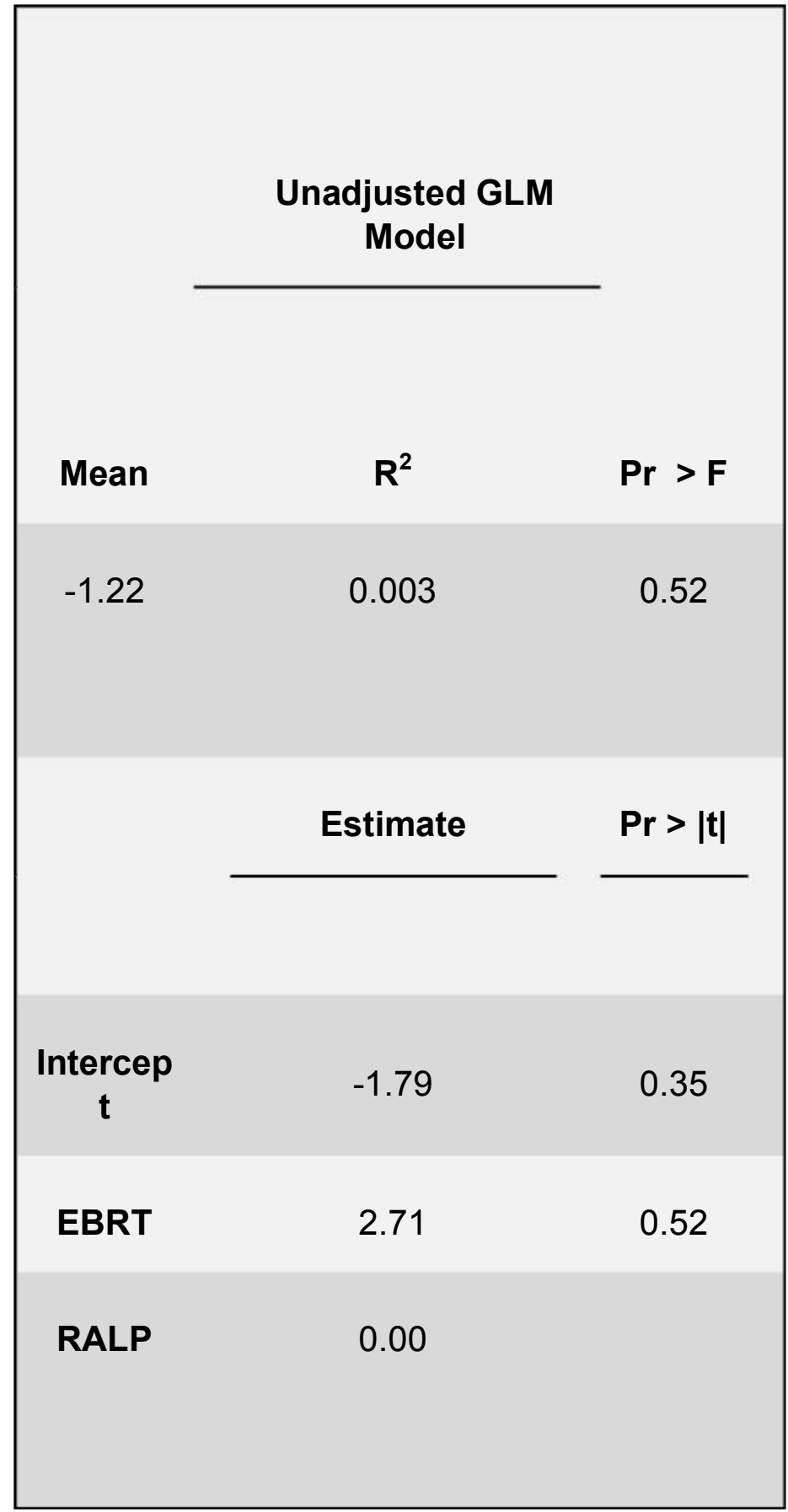


Table 5 shows the reported values of the fully adjusted GLM model that included possible confounding variables. The variables that were found to confound the association (to have an effect on change in urinary EPIC score independent of treatment group) of study variables were cardiac disease, arterial disease, age, body mass index (BMI), smoking, hypertension, and hormone treatment. None of these variables were found to modify the association (act in association with treatment group to affect urinary EPIC score) of study variables. The F-value for the fully adjusted GLM model was 0.0076, indicating a good fitting model (see table 5). The $\mathrm{R}^{2}$ value generated by this analysis was 0.18 . This indicates that the treatment groups and all of the confounding variables explain $18 \%$ of the variation in urinary change for all the patients included in this analysis (see table 5). EBRT had 4.45 greater increase in urinary change score than RALP, indicating a worsening in urinary function from baseline (as positive values represent a worsening in function) (see table 5). This association is not statistically significant with a p-value of 0.40 . Thus, no statistically significant change was detected between urinary score change for EBRT and RALP. The mean value of all patients included in the GLM procedure was -0.81 (see table 5). As this value is negative, it shows that there was a general improvement of urinary function in men treated for prostate cancer with either treatment.

\section{Table 5}

Reported Values from the Urinary Function Fully Adjusted GLM Model

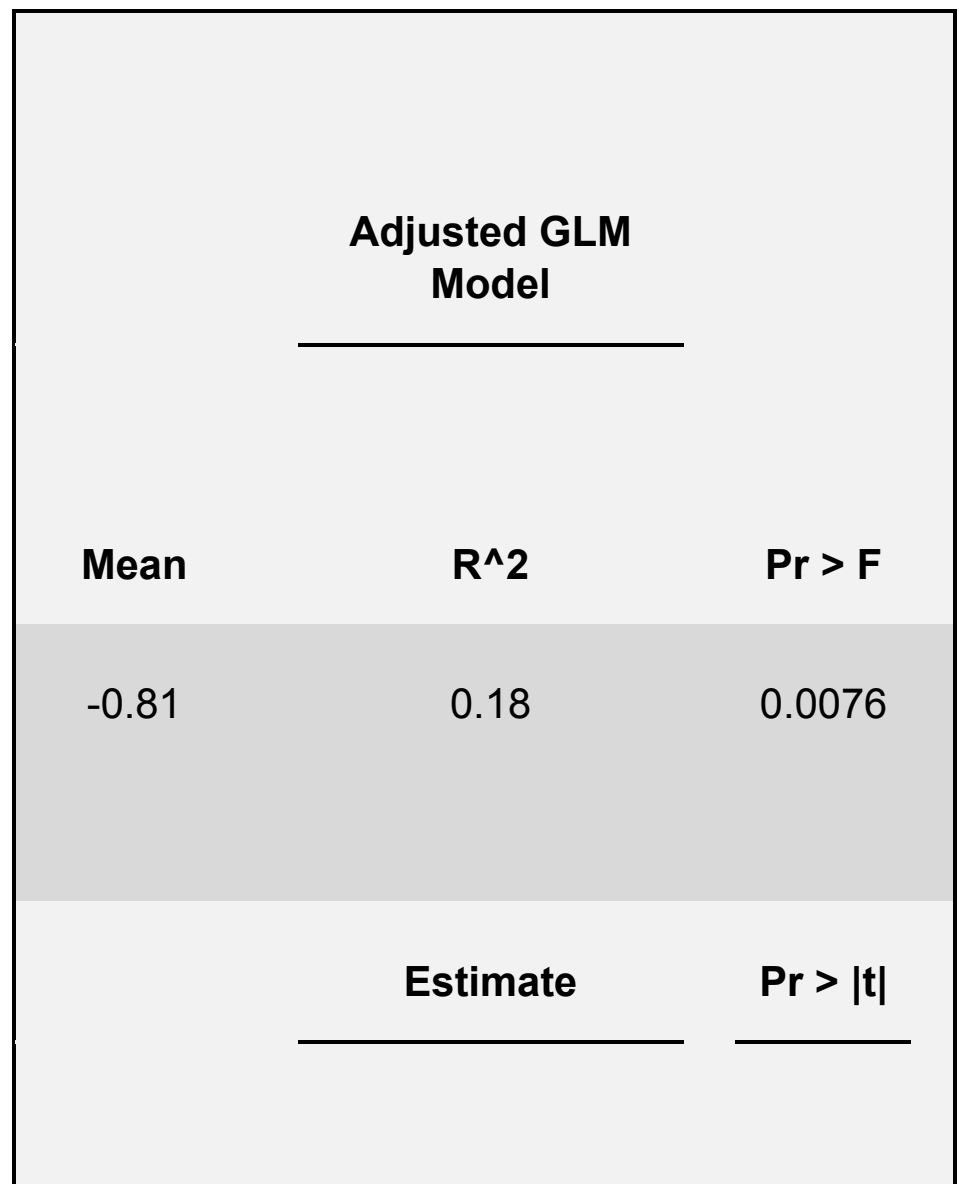




\begin{tabular}{|ccc|} 
Intercept & 10.19 & 0.61 \\
EBRT & 4.45 & 0.41 \\
RALP & 0.00 & \\
& & \\
\hline
\end{tabular}

Figure 6 shows the unadjusted GLM model of change in sexual function from pre-treatment to two years post treatment. The F-value reported in Table 6 for this model was 0.012 indicating the model was a good fit. The $\mathrm{R}^{2}$ value is 0.05 , indicating that $5 \%$ of the variation in change sexual function from pre-treatment to 2 years is explained by type of treatment (see table 6). The difference in sexual function for patients undergoing EBRT was -11.9, which was statistically different $(\mathrm{p}=0.01)$ (see table 6). As negative numbers represent an improvement in sexual function from baseline, this indicates that EBRT patients had better sexual function than RALP patients. 
Fig 6. Distribution of Sexual EPIC Score Change

In this figure, o denotes baseline. As two year scores were subtracted from pre-treatment scores, negative scores indicate improvement from initial urinary function and positive scores represent a worsening from initial urinary function.

*Indicates a statistically significant difference $(\mathrm{p}<0.05)$

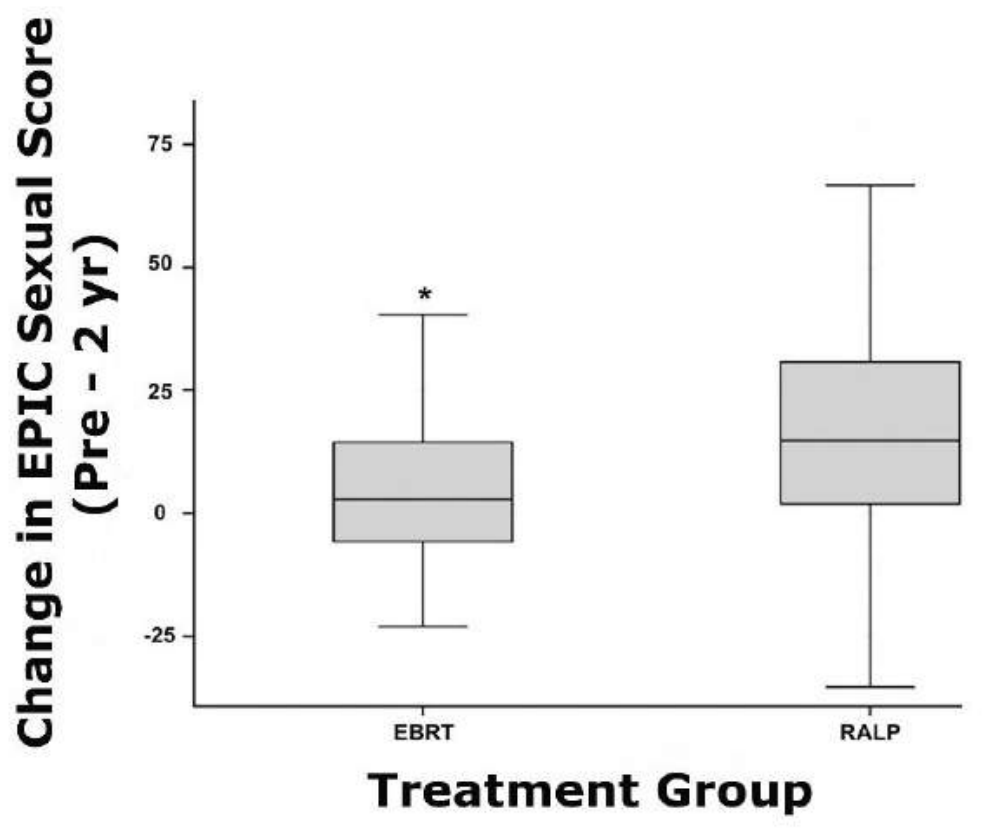




\section{Table 6}

Reported Values from Sexual Function GLM Unadjusted Model

\begin{tabular}{|c|c|c|}
\hline & $\begin{array}{c}\text { Unadjusted GLM } \\
\text { Model }\end{array}$ & \\
\hline Mean & $R^{\wedge} 2$ & $\operatorname{Pr}>\mathrm{F}$ \\
\hline \multirow[t]{2}{*}{15.94} & 0.052 & 0.012 \\
\hline & Estimate & $\operatorname{Pr}>|t|$ \\
\hline $\begin{array}{l}\text { Intercep } \\
\mathbf{t}\end{array}$ & 18.34 & $<.0001$ \\
\hline EBRT & -11.88 & 0.012 \\
\hline RALP & 0.00 & \\
\hline
\end{tabular}


Table 7

Reported Values from Sexual Function Fully Adjusted GLM Model

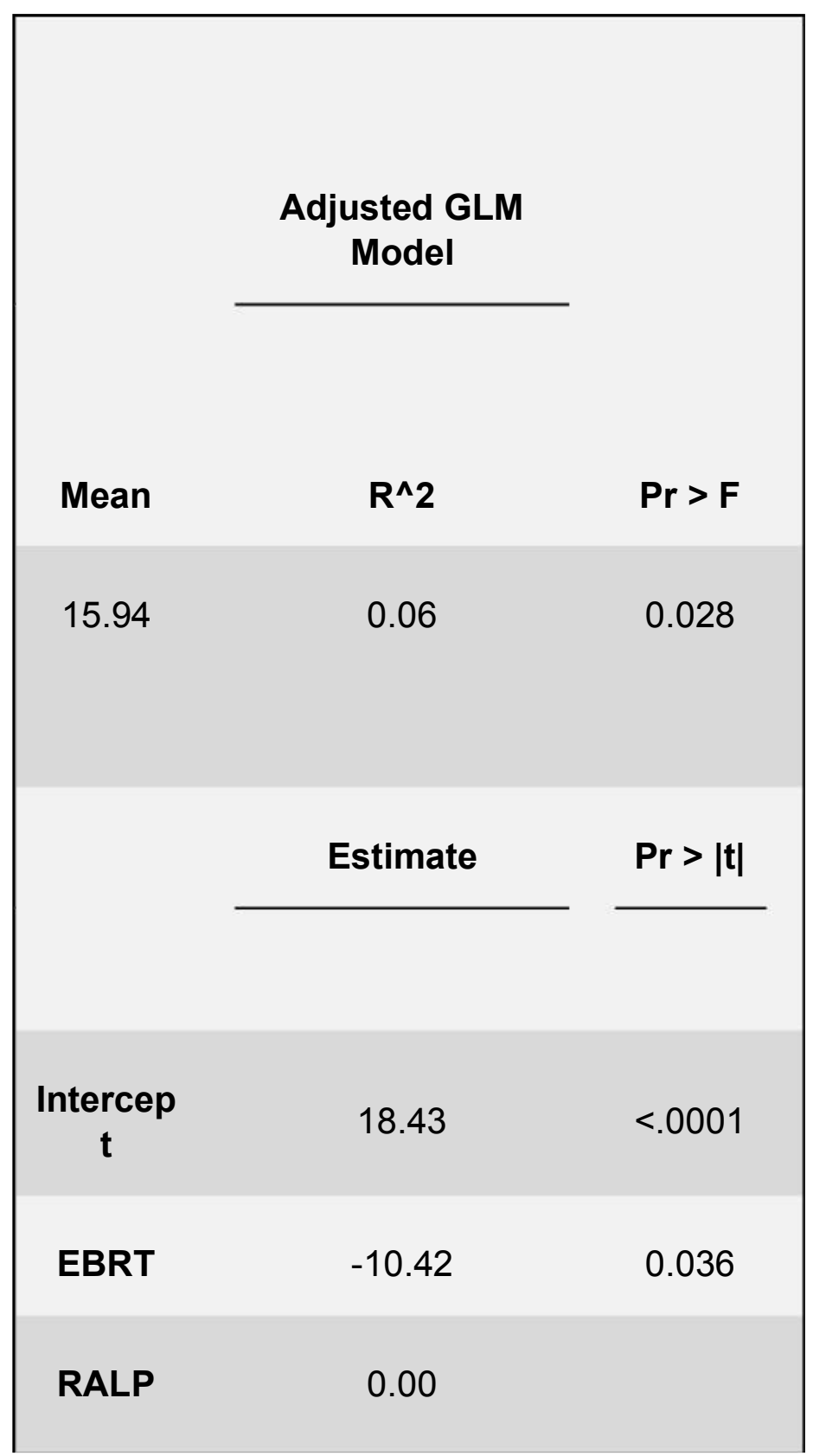

The fully adjusted GLM procedure included hormone treatment in the analysis, as it was found to confound the relationship between treatment group and change in EPIC sexual function score. However, it was found that hormone treatment did not modify the association (act in conjunction with type of treatment) to affect change in EPIC score. The $\mathrm{F}$ value for the fully adjusted GLM model shown in Table 7 was 0.028 , demonstrating a model of good fit. The $\mathrm{R}^{2}$ value was 0.06 , which indicates that treatment group can explain $6 \%$ of the variation in change in sexual function from 
baseline to two years (see table 7). The difference in change between the EBRT patients and the RALP patients was -10.4, which is a statistically significant difference $(p=0.04)$ (see table 7). As negative numbers represent an increase in function, EBRT patients have better sexual function than RALP patients.

\section{Discussion}

This study compared External Beam Radiation Therapy (EBRT) to Robot Assisted Laparoscopic Prostatectomy (RALP) in terms of urinary and sexual function change from pretreatment. The purpose of this comparison was to determine if either treatment provided better outcomes for patients. It was hypothesized that RALP patients would have better improvement in urinary function, and EBRT patients would have better improvement in sexual function.

The study's results found no significant difference between the treatments for change in urinary function at the two-year time point. This indicates that neither treatment is superior at preserving urinary function. The mean value of all of the patients included in the analysis of urinary function was negative. This indicates that, in general, all patients who undergo treatment for prostate cancer experience improved urinary function from before their initial treatment. This study's finding of no significant difference between urinary function change and treatment groups does not coincide with a study performed by Chien in 2017. This study found that urinary function was significantly worse in RALP patients compared to other treatments such as EBRT (Chien et al. 520). As there are still few studies that compare RALP to EBRT these conflicting findings cannot be reconciled.

A significant difference was found in the change in sexual function scores between treatment groups. The EBRT patients had a difference in change of -10.4 from the RALP patients. As negative numbers indicate an improvement in function, EBRT patients' sexual function improved when compared to RALP patients $(\mathrm{p}=0.04)$. This indicates that EBRT is the superior treatment in the preservation of sexual function. These findings agree with Chein's 2017 study that found EBRT patients had better sexual function when compared to RALP patients.

This study has limitations that affect the conclusions that are drawn. As this study was performed with self-report EPIC questionnaires, there is the chance that the answers the subjects provided are not an accurate representation of their urinary or sexual function. There was also a much smaller sample size of EBRT patients compared to RALP patients, largely due to extensive missing data in pre-treatment scores for patients treated with EBRT. The missing data likely results from differences in EPIC packet distribution across sites where treatment is being delivered. The Research Department is located at the main campus, the same site where RALP patients receive treatment. This allows more effective pre-treatment packet delivery to RALP patients when compared to patients being treated at the satellite EBRT clinic, where the Research Department has much less direct influence over packet delivery. This study also relied heavily on data in patient charts for assessment of cofounding variables. If these charts were not updated or did not contain the information gathered by this study, the effect of these cofounding variables determined in this study could be inaccurate. As was mentioned, there was extensive 
missing data for two variables: erectile aid use and race. A study that was designed to collect this information in a more reliable way, rather than relying on information included in a patient's chart, would be able to assess confounding variables to a more accurate degree. The diversity of patients is also a limitation. The racial demographic of subjects included in this study was comprised almost entirely of white men, which makes it difficult for the findings to be representative of the entire population of men with prostate cancer. In addition to race, this study was unable to include socioeconomic status and other influences such as access to health care facilities in the analysis. This severely limits the implications of the study's findings when applied to larger populations, since these are important indicators in health outcomes and could have confounded the results of this study. A study that examined a group of more diverse subjects, and included other determinants of health outcomes such as socioeconomic status would be able to draw more extensive conclusions.

This is one of the first studies that compares RALP to other treatments. More studies that compare RALP to other treatments are needed to assess which treatment will best suit patient needs. As use of erectile aids was not able to be included in this analysis due to extensive missing data, studies that analyze the role of erectile aid use in sexual function change after prostate cancer treatment should be conducted to evaluate how erectile aid use affects the change in sexual function in relation to different treatment types.

\section{Conclusion}

Health related quality of life in regards to urinary and sexual function varies by treatment. This study supports that EBRT is a better therapy for preserving sexual function, and demonstrates that either treatment will preserve urinary function to a similar degree. These findings will supplement the growing information about how different prostate cancer treatments will affect a patient's quality of life after treatment, thus providing the option for treatments to be specified to a patient's needs. The more patients can be informed about their health care choices the more they can understand the implications and consequences of their choices to make a decision about treatment that is best for them. Studies such as this that compare RALP and EBRT within a larger and more diverse population are needed to support and add to this study's finding, thereby achieving a greater understanding of how these treatments affect quality of life after treatment.

\section{Acknowledgements}

There are many wonderful people who helped me through this rewarding process, and I appreciate the opportunity to formally thank them now. I would like to thank Dr. Carrie McCurdy for agreeing to be my Primary Thesis Advisor. Thank you for your guidance that allowed me to find the most incredible resources that were invaluable to the development of this project. Thank you to Dr. David DiMarco for helping me navigate the nuances of clinical research for prostate cancer. I appreciate your expertise in this field. Thank you to Dr. Kelly Sutherland for being a wonderful CHC advisor, and for agreeing to be on my Thesis Committee. I have greatly valued your guidance in my time here at the University of Oregon. I am so grateful 
to Gerald Rouleau, a former OUI intern and a wonderful statistical analyst, who saved my sanity by agreeing to help me with the statistical analysis. The analysis you conducted was invaluable to the validity and importance of this project. Thank you Jon Runyeon, for kindly offering to let me do an abdominal prosection on a cadaver in the anatomy lab so I could understand the prostate anatomy on a more in-depth level. The experience helped me understand the significance of the abdominal anatomy in this project. Perhaps I am most appreciative to Stephanie Kerns and Renee Podesta, who must have decided that I was sassy enough to get along with and chose to give me the opportunity of being an intern at OUI. You have both been so helpful and encouraging as I have worked to pull this project together, and I am so grateful. Thank you to Martin Allums, who created the fabulous illustrations that made this project look much more professional than I could have ever imagined. I look forward to the day when every medical textbook I open is bursting with your illustrations. Finally, I would like to thank my parents Liz and Doug Fischer. Your support and never-ending encouragement has always allowed me to do way more than I ever should, and kept me sane while I muddled through it. In the end I know that I am Julia Pauline Fischer, and I am loved very much. Thank you for being my parents, I love you.

\section{Works Cited}

Barré, Christian. "Open Radical Retropubic Prostatectomy.” European Urology, vol. 52, no. 1, 2007, pp. 71-80, doi:10.1016/j.eururo.2006.11.057.

Chien, Gary W., et al. "Health-Related Quality of Life Outcomes from a Contemporary Prostate Cancer Registry in a Large Diverse Population.” BJU International, vol. 120, no. 4,2017, doi:10.1111/bju.13843.

Coelho, Rafael F., et al. "Retropubic, Laparoscopic, and Robot-Assisted Radical Prostatectomy: A Critical Review of Outcomes Reported by High-Volume Centers.” Journal of Endourology, vol. 24, no. 12, 2010, pp. 2003-15, doi:10.1089/end.2010.0295.

Coyne, Karin S., et al. "The Impact of Overactive Bladder, Incontinence and Other Lower Urinary Tract Symptoms on Quality of Life, Work Productivity, Sexuality and Emotional Well-Being in Men and Women: Results from the EPIC Study." BJU International, vol. 101, no. 11, June 2008, pp. 1388-95, doi:10.1111/j.1464-410X.2008.07601.x.

Donovan, Jenny L., et al. "Patient-Reported Outcomes after Monitoring, Surgery, or Radiotherapy for Prostate Cancer." New England Journal of Medicine, vol. 375, no. 15,2016, pp. 1425-37, doi:10.1056/NEJMoa1606221.

Edge, Stephen B., and Carolyn C. Compton. "The American Joint Committee on Cancer: The 7th Edition of the AJCC Cancer Staging Manual and the Future of TNM." Annals of Surgical Oncology, vol. 17, no. 6, 2010, pp. 1471-74, doi:10.1245/s10434-010-0985-4.

Fowler, Floyd J., et al. "Comparison of Recommendations by Urologists and Radiation Oncologists for Treatment of Clinically Localized Prostate Cancer.” JAMA, vol. 283, no. 24, 2000, p. 3217, doi:10.1001/jama.283.24.3217.

Frank, Steven J., et al. “An Assessment of Quality of Life Following Radical Prostatectomy, High 
Dose External Beam Radiation Therapy and Brachytherapy Iodine Implantation as Monotherapies for Localized Prostate Cancer.” The Journal of Urology, vol. 177, no. 6, 2007, pp. 2151-56, doi:10.1016/j.juro.2007.01.134.

Haas, Gabriel P., et al. "The Worldwide Epidemiology of Prostate Cancer: Perspectives from Autopsy Studies.” The Canadian Journal of Urology, vol. 15, no. 1, 2008, pp. 3866-71.

Heidenreich, Axel, et al. "EAU Guidelines on Prostate Cancer." European Urology, vol. 53, no. 1, 2008, pp. 68-80, doi:10.1016/j.eururo.2007.09.002.

Humphrey, Peter A. "Gleason Grading and Prognostic Factors in Carcinoma of the Prostate." Modern Pathology, vol. 17, no. 3, 2004, pp. 292-306, doi:10.1038/modpathol.3800054.

Lee, Chung, et al. "Demonstration of the Role of Prostate-Specific Antigen in Semen Liquefaction by Two-Dimensional Electrophoresis." Journal of Andrology, vol. 10, no. 6, 1989, pp. 432-38, doi:10.1002/j.1939-4640.1989.tbo0134.x.

Lepor, Herbert. "Selecting Candidates for Radical Prostatectomy.” Reviews in Urology, vol. 2, no. 3,2000 , pp. 182-89.

Litwin, Mark S., et al. "Quality of Life after Surgery, External Beam Irradiation, or Brachytherapy for Early-Stage Prostate Cancer." Cancer, vol. 109, no. 11, 2007, pp. 2239-47, doi:10.1002/cncr.22676.

Miller, David C., et al. "Long-Term Outcomes among Localized Prostate Cancer Survivors: Health-Related Quality-of-Life Changes after Radical Prostatectomy, External Radiation, and Brachytherapy." Journal of Clinical Oncology, vol. 23, no. 12, 2005, pp. 2772-8o, doi:10.1200/JCO.2005.07.116.

Miyamoto, Hiroshi, et al. "Androgen Deprivation Therapy for Prostate Cancer: Current Status and Future Prospects." The Prostate, vol. 61, no. 4, 2004, pp. 332-53, doi:10.1002/pros.20115.

Penson, David F., et al. "5-Year Urinary and Sexual Outcomes After Radical Prostatectomy: Results From the Prostate Cancer Outcomes Study." The Journal of Urology, vol. 179, no. 5, 2008, pp. S40-44, doi:10.1016/j.juro.2008.03.136.

Potosky, Arnold L., et al. "Five-Year Outcomes after Prostatectomy or Radiotherapy for Prostate Cancer: The Prostate Cancer Outcomes Study.” Journal of the National Cancer Institute, vol. 96, no. 18, 2004, pp. 1358-67, doi:10.1093/jnci/djh259.

Schreiber, David, et al. "Clinical and Biochemical Outcomes of Men Undergoing Radical Prostatectomy or Radiation Therapy for Localized Prostate Cancer.” Radiation Oncology Journal, vol. 33, no. 1, 2015, pp. 21-28, doi:10.3857/roj.2015·33.1.21.

Shah, Rajal B. "Current Perspectives on the Gleason Grading of Prostate Cancer." Archives of Pathology \& Laboratory Medicine, vol. 133, no. 11, 2009, pp. 1810-16, doi:10.1043/15432165-133.11.1810.

Smith, Robert A., et al. "Cancer Screening in the United States, 2009: A Review of Current 
American Cancer Society Guidelines and Issues in Cancer Screening." CA: A Cancer Journal for Clinicians, vol. 59, no. 1 2009, pp. 27-41, doi:10.3322/caac.20008.

Stenman, Ulf-Håkan, et al. "Prostate-Specific Antigen.” Seminars in Cancer Biology, vol. 9, no. 2,1999, pp. 83-93, doi:10.1006/scbi.1998.0086.

Talcott, James A., et al. "Patient-Reported Impotence and Incontinence after Nerve-Sparing Radical Prostatectomy." Journal of the National Cancer Institute, vol. 89, no. 15, 1997, pp. 1117-23, doi:10.1093/JNCI/89.15.1117.

Walsh, Patrick C., et al. "Radical Prostatectomy with Preservation of Sexual Function: Anatomical and Pathological Considerations." The Prostate, vol. 4, no. 5, 1983, pp. 473-85, doi:10.1002/pros.2990040506.

Wei, John T., et al. "Development and Validation of the Expanded Prostate Cancer Index Composite (EPIC) for Comprehensive Assessment of Health-Related Quality of Life in Men with Prostate Cancer.” Urology, vol. 56, no. 6, 2000, pp. 899-905, doi:10.1016/Soo9o4295(00)00858-X. 